\title{
Wideband Unit-Cell based on Liquid Crystals for Reconfigurable Reflectarray Antennas in F-band
}

\author{
G. Perez-Palomino, J. A. Encinar \\ M. Barba
}

R. Dickie, P. Baine, R. Cahill

\author{
R. Florencio, R. R. Boix
}

\begin{abstract}
A reconfigurable reflectarray-cell has been designed to provide a large tunable phase range in the frequency band 117$130 \mathrm{GHz}$. The unit-cell comprises three parallel dipoles printed on a quartz-wafer and a tunable liquid crystal (LC) placed on a cavity between the dipoles and a ground plane. The simulation results show a tunable phase-shift in a range larger than $\mathbf{3 0 0}$ degree for a $10 \%$ bandwidth and low sensitivity to the angle of incidence.
\end{abstract}

\section{INTRODUCTION}

At present, there is a significant interest in reconfigurable reflectarray antennas because of their relatively low cost and ease of construction. Beam-steering capabilities can be obtained by electronically controlling the phase-shift on the reflectarray (RA) cells. The anisotropic properties of liquid crystals (LC) can be used to dynamically adjust the phase on each RA-cell. If a $\mathrm{LC}$ is used as an active medium between a resonant patch and the ground plane, when a bias voltage is applied, the molecules are reoriented in the direction of the electric field, producing an increase of the dielectric constant at macroscopic level and a variation of the phase-shift in the RAelement.

The recent interest in applications at frequencies above 100 $\mathrm{GHz}$, such as spectroscopy, atmospheric remote sensing or security, makes the use of Liquid Crystals (LCs) more attractive, because conventional phase-shifters are not usable at these frequencies at the present state of the technology. Several unit-cells based on a resonant patch above a LC cavity have been realized and characterized at $77 \mathrm{GHz}$ [1] and above 100 $\mathrm{GHz}$ [2]. However, these cells suffer from limitations in bandwidth, phase range and losses. These limitations have been overcome by using a multi-resonant architecture based on three parallel dipoles over a LC layer [3], as shown in Fig. 1. A unitcell at $35 \mathrm{GHz}$ was designed to maximize the phase range and the bandwidth and to minimize the reflection losses [3]. However, the angle of incidence was not considered in the design [3], obtaining thus a good electrical behavior only in a limited angular range for the incident field.

In this paper, a wideband LC-reflectarray cell has been designed using a new LC (GT3-23001) manufactured by Merck KGaA to improve the performance in millimeter-wave frequencies. The LC has been experimentally characterized in F-band by Queen's University Belfast (QUB). These results have been used to design a reflectarray element to improve the bandwidth, the tunable phase range and to reduce the sensitivity of the phase response with respect to the angle of incidence and manufacturing tolerances.

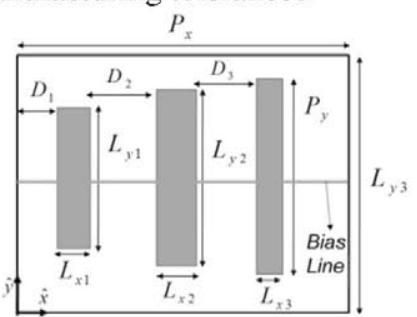

(a)

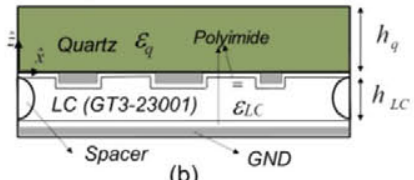

(b)

Figure 1. Reconfigurable reflectarray-Cell based on LC and three parallel dipoles. Top (a) and lateral (b) views.

\section{DESIGN OF THE CELL}

The configuration of the RA-cell for broadband operation is based on three parallel dipoles printed on the same side of a quartz-wafer $\left(\varepsilon_{q}=3.78, \tan \delta=0.002\right)$ of thickness $0.55 \mathrm{~mm}$. The LC GT3-23001 from Merck is enclosed in a cavity bounded by the ground plane and the printed dipoles, whose height is fixed by $125 \mu \mathrm{m}$ separators. Both the ground plane and the quartz with the printed dipoles are covered with a thin polyimide film (rubbing layer), whose electromagnetic effects may be neglected because of the small thicknesses ( $\sim 100 \mathrm{~A}$ ). The electrical parameters of the LC provided by Merck at $19 \mathrm{GHz}$ are: $\varepsilon_{\mathrm{r} / /}=3.3, \tan \delta_{/ /}=0.0038, \varepsilon_{\mathrm{r} \perp}=2.5, \tan \delta_{\perp}=$ 0.0143 . Theses parameters were also measured at QUB using a cavity backed by a resonant FSS [4] and the response was simulated by CST ${ }^{\circledR}[5]$ in the frequency band $140-165 \mathrm{GHz}$. The LC values employed in the numerical model were $\varepsilon_{\mathrm{r} / /}=3.25, \tan \delta_{/ /}=0.015, \varepsilon_{\mathrm{r} \perp}=2.47, \tan \delta_{\perp}=0.02$, which are very 
similar to the dielectric constant but higher in loss, compared to those reported by the manufacturer at $19 \mathrm{GHz}$. It was checked that this new LC provides better tunability and lower losses at $140 \mathrm{GHz}$ than other conventional LC mixtures, such as BL037.

The design criteria given in [3] were first applied to adjust the geometrical parameters of the unit-cell shown in Fig. 1 in order to extend the bandwidth and phase range at normal incidence. The reflectarray cell was analyzed by in-house software tool based on Spectral Domain MoM [6], which has been validated by comparing the results with those obtained by CST [5]. In this first iteration, considering the nominal properties of the $\mathrm{LC}$, a phase range of $360^{\circ}$ was obtained in a bandwidth (defined by an error of $\pm 20^{\circ}$ [3]) from 117 to 130 $\mathrm{GHz}(10.5 \%)$. The resulting dimensions were considered as a starting point to design the cell taking into account the angle of incidence. The design is carried out making a sequential adjustment of the parameters, basically reducing the period of the cell and increasing the widths of the dipoles. The new dimensions, see Table 1, provide a lower sensitivity to the angle of incidence and dimensions, but the range in phase is slightly reduced.

\section{NUMERICAL RESULTS}

The amplitude (losses) and phase of the reflection coefficient have been computed for the extreme and intermediate values of the LC permittivity and loss tangent measured at $140 \mathrm{GHz}$. Figure 2 shows the simulated results for three angles of incidence. The bandwidth remains similar over a large angular range, but the phase range is slightly reduced at some frequencies. The $30 \mu \mathrm{m}$ thick lines used to introduce the bias voltage, see Fig. 1, have been modeled for different angles of incidence using CST and their effects are negligible. As a result of the higher measured losses, there is an increase in losses between 1 and $2 \mathrm{~dB}$, with respect to those obtained for the nominal loss-tangent of the LC. These losses can be reduced by using a new generation of $\mathrm{LC}$ engineered to improve the performances in millimeter-wave frequencies. The proposed reflectarray cell can be used in low cost reconfigurable antennas beyond $100 \mathrm{GHz}$.

\section{ACKNOWLEDGEMENT}

This work has been supported by "Fundacion Caja Madrid", by the Spanish Ministry of Science and Innovation (projects TEC2010-17567 and Consolider-Ingenio CSD2008-00068 "TERASENSE") and by the European Space Agency (ESA).

\section{REFERENCES}

[1] R. Moessinger, J. Marin, S. Freese, S. Mueller, A. Manabe, R. Jakoby, "77 GHz reconfigurable reflectarray with nematic liquid crystal," Proc. 2nd EuCAP, Edinburgh, Nov. 2007.

[2] W. Hu, R. Cahill, J. A. Encinar, R. Dickie, H. Gamble, V. Fusco, and N. Grant, "Design and measurement of reconfigurable millimeter wave reflectarray cells with nematic liquid crystal," IEEE Trans. Antennas Propag., vol. 56, no. 10, pp. 3112-3117, Oct. 2008.

[3] G. Perez-Palomino, J. A. Encinar, M. Barba, E. Carrasco, "Desion and Evaluation of Multi-Resonant Unit-Cells Based on Liquid Crystals for Reconfigurable Reflectarrays," IET. Microw. Antennas Propagat, in press.

[4] R. Simms, R. Dickie, R. Cahill, N. Mitchell, H. Gamble, V. Fusco, "Measurement of Electromagnetic Properties of Liquid Crystals at 300
GHz Using a Tunable FSS," 31st ESA Workshop on Antennas for Space Applications, European Space Agency, Holland, Oct. 2010.

[5] CST Microwave Studio(ß). [Online]. Available: http://www.cst.com

[6] R. Florencio, R. R Boix, J.A. Encinar, "Efficient analysis of multiresonant periodic structures for the improved analysis and design of reflectarray antennas", 6th EUCAP, Prague, March 2012.

TABLE I. DIMENSIONS FOR THE DESIGNED CELL

\begin{tabular}{|c|c|}
\hline Variable & Value $(\mathbf{m m})$ \\
\hline Px, Py, hLC, hq & $1.05,0.9,0.125,0.55$ \\
\hline Ly1, Ly2, Ly3, & $0.52,0.55,0.60$ \\
\hline Lx1, Lx2, Lx3 & $0.14,0.14,0.14$ \\
\hline D1, D2, D3 & $0.115,0.215,0.15$ \\
\hline
\end{tabular}
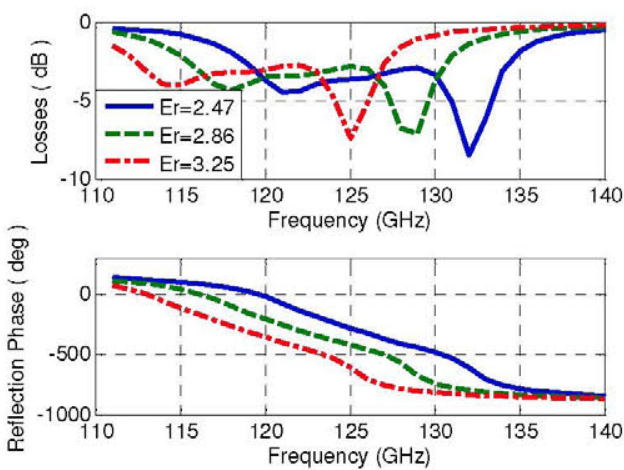

(a)
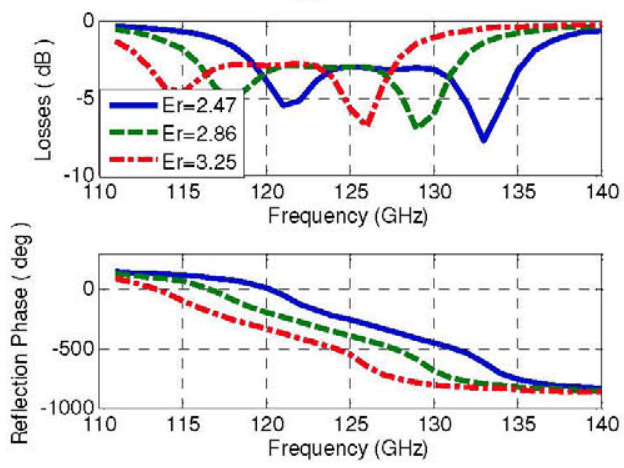

(b)
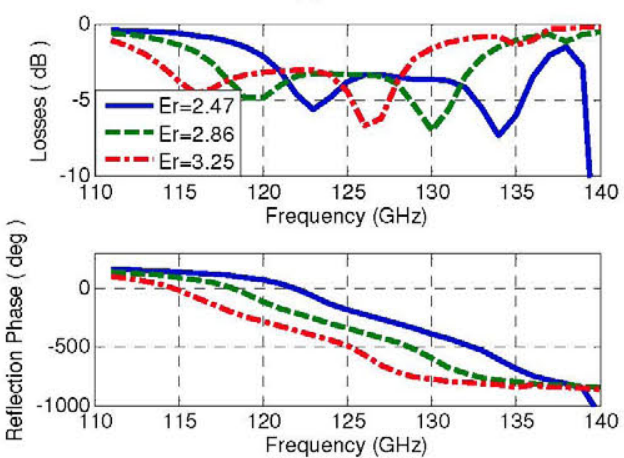

(c)

Figure 2. Amplitude and phase of the reflection coefficient for the 3-dipole RA-cell at normal incidence (a); $\theta=30^{\circ}, \varphi=60^{\circ}(\mathrm{b})$; and $\theta=45^{\circ}, \varphi=90^{\circ}$ (c). 